ARTICLE

\title{
X-ray studies bridge the molecular and macro length scales during the emergence of $\mathrm{CoO}$ assemblies
}

Lukas Grote (1D) 1,2,11, Cecilia A. Zito (1D 1,3,11, Kilian Frank (DiD) 4,11, Ann-Christin Dippel (iD 2 ${ }^{2}$ Patrick Reisbeck ${ }^{4}$, Krzysztof Pitala (1) 5,6, Kristina O. Kvashnina (1) 7,8, Stephen Bauters ${ }^{7,8}$, Blanka Detlefs (1) 9, Oleh Ivashko (1) 2, Pallavi Pandit ${ }^{2}$, Matthias Rebber (D) 1,10, Sani Y. Harouna-Mayer (1) 1,10, Bert Nickel ${ }^{4}$ \& Dorota Koziej (D) 1,10凶

The key to fabricating complex, hierarchical materials is the control of chemical reactions at various length scales. To this end, the classical model of nucleation and growth fails to provide sufficient information. Here, we illustrate how modern $\mathrm{X}$-ray spectroscopic and scattering in situ studies bridge the molecular- and macro- length scales for assemblies of polyhedrally shaped $\mathrm{CoO}$ nanocrystals. Utilizing high energy-resolution fluorescence-detected X-ray absorption spectroscopy, we directly access the molecular level of the nanomaterial synthesis. We reveal that initially $\mathrm{Co}(\mathrm{acac})_{3}$ rapidly reduces to square-planar $\mathrm{Co}(\mathrm{acac})_{2}$ and coordinates to two solvent molecules. Combining atomic pair distribution functions and small-angle $\mathrm{X}$-ray scattering we observe that, unlike a classical nucleation and growth mechanism, nuclei as small as $2 \mathrm{~nm}$ assemble into superstructures of $20 \mathrm{~nm}$. The individual nanoparticles and assemblies continue growing at a similar pace. The final spherical assemblies are smaller than $100 \mathrm{~nm}$, while the nanoparticles reach a size of $6 \mathrm{~nm}$ and adopt various polyhedral, edgy shapes. Our work thus provides a comprehensive perspective on the emergence of nano-assemblies in solution.

\footnotetext{
${ }^{1}$ University of Hamburg, Institute for Nanostructure and Solid-State Physics, Center for Hybrid Nanostructures, Hamburg, Germany. ${ }^{2}$ Deutsches ElektronenSynchrotron DESY, Hamburg, Germany. ${ }^{3}$ São Paulo State University UNESP, São José do Rio Preto, Brazil. ${ }^{4}$ Ludwig-Maximilians-Universität München, Faculty of Physics and Center for NanoScience (CeNS), Munich, Germany. ${ }^{5}$ AGH, University of Science and Technology, Faculty of Physics and Applied Computer Science, Krakow, Poland. ${ }^{6}$ Academic Center for Materials and Nanotechnology, AGH University of Science and Technology, Krakow, Poland. ${ }^{7}$ The Rossendorf Beamline at the European Synchrotron Radiation Facility ESRF, Grenoble, France. ${ }^{8}$ Helmholtz-Zentrum Dresden-Rossendorf (HZDR), Institute of Resource Ecology, Dresden, Germany. ${ }^{9}$ European Synchrotron Radiation Facility ESRF, Grenoble, France. ${ }^{10}$ The Hamburg Centre for Ultrafast Imaging, Hamburg, Germany. ${ }^{11}$ These authors contributed equally: Lukas Grote, Cecilia A. Zito, Kilian Frank. ${ }^{凶}$ email: dorota.koziej@physnet.uni-hamburg.de
} 
W ith the emerging demand for materials with complex morphological and structural properties, understanding their formation in solution continues to be a major challenge. Most syntheses of colloidal nanoparticles rely on classical crystallization and growth by monomer addition from supersaturated media, which enables the rational synthesis of nanocrystals ${ }^{1-7}$. The recently discovered nonclassical formation pathways involving the assembly of building blocks into distinctive superstructures have opened up completely new ways of complex nanomaterial design ${ }^{8-11}$. Different mechanisms exist within the scope of these nonclassical pathways ${ }^{10,12,13}$. Besides the oriented assembly of nanocrystals into mesocrystals ${ }^{8,14,15}$, the formation of polycrystalline, unoriented assemblies from distinct primary structures ${ }^{9,16-20}$ is a major yet rather unexplored branch (see Cölfen et al. $2019^{10}$, Fig. 1d). To date, nonclassical nucleation is still far from reaching the level of control established for classical growth pathways. This is by no means a surprise, since the fundamental phenomena cannot be easily generalized, and the existing theory merely provides an overview of possible pathways $10,21,22$. The major experimental difficulties to provide a unique description arise from the fact that we need to study the complex chemical and structural changes on multiple length scales.

To date, synchrotron-based methods such as X-ray absorption spectroscopy and powder X-ray diffraction (PXRD) ${ }^{23-31}$ are routinely combined to study colloidal reactions. However, to follow chemical and electronic changes in complex reactions in solution with high sensitivity and at low concentrations ${ }^{32-35}$, more advanced spectroscopic techniques, such as high energyresolution fluorescence-detected X-ray absorption near edge structure (HERFD-XANES) are needed. Compared with conventional X-ray absorption spectroscopy, the higher energy resolution of HERFD-XANES makes it sensitive to subtle changes in the local chemical environment of the absorbing atom.

Complementary structural information can be obtained from the pair distribution function (PDF) analysis of high-energy in situ X-ray total scattering. This method takes into account both the Bragg scattering from crystalline and the diffuse scattering from amorphous phases. It results in a real-space representation of the interatomic distances of all constituents in a reaction mixture. In this manner, local ordering during the formation of nuclei, the initial growth of nanocrystals, as well as the restructuring of solvent molecules at the surface of nanoparticles were revealed ${ }^{36-39}$. Self-assembly of building blocks into superstructures can be probed with small-angle X-ray scattering $(\mathrm{SAXS})^{40-42}$. This way, length scales all the way from atomic to macroscopic are accessible via X-ray techniques.

Here, we uniquely combine HERFD-XANES, PDF, and SAXS to study the emergence of $\mathrm{CoO}$ nanoassemblies, a noticeable example for a nonclassically crystallized nanostructure. In general, cobalt oxide has applications in lithium ion 43,44 and lithium oxygen batteries ${ }^{45}$, as a catalyst for electrochemical ${ }^{46,47}$ and for solar water splitting ${ }^{4-52}$. Cobalt precursors under solvothermal conditions ${ }^{53}$ show a complex redox behavior that can lead to
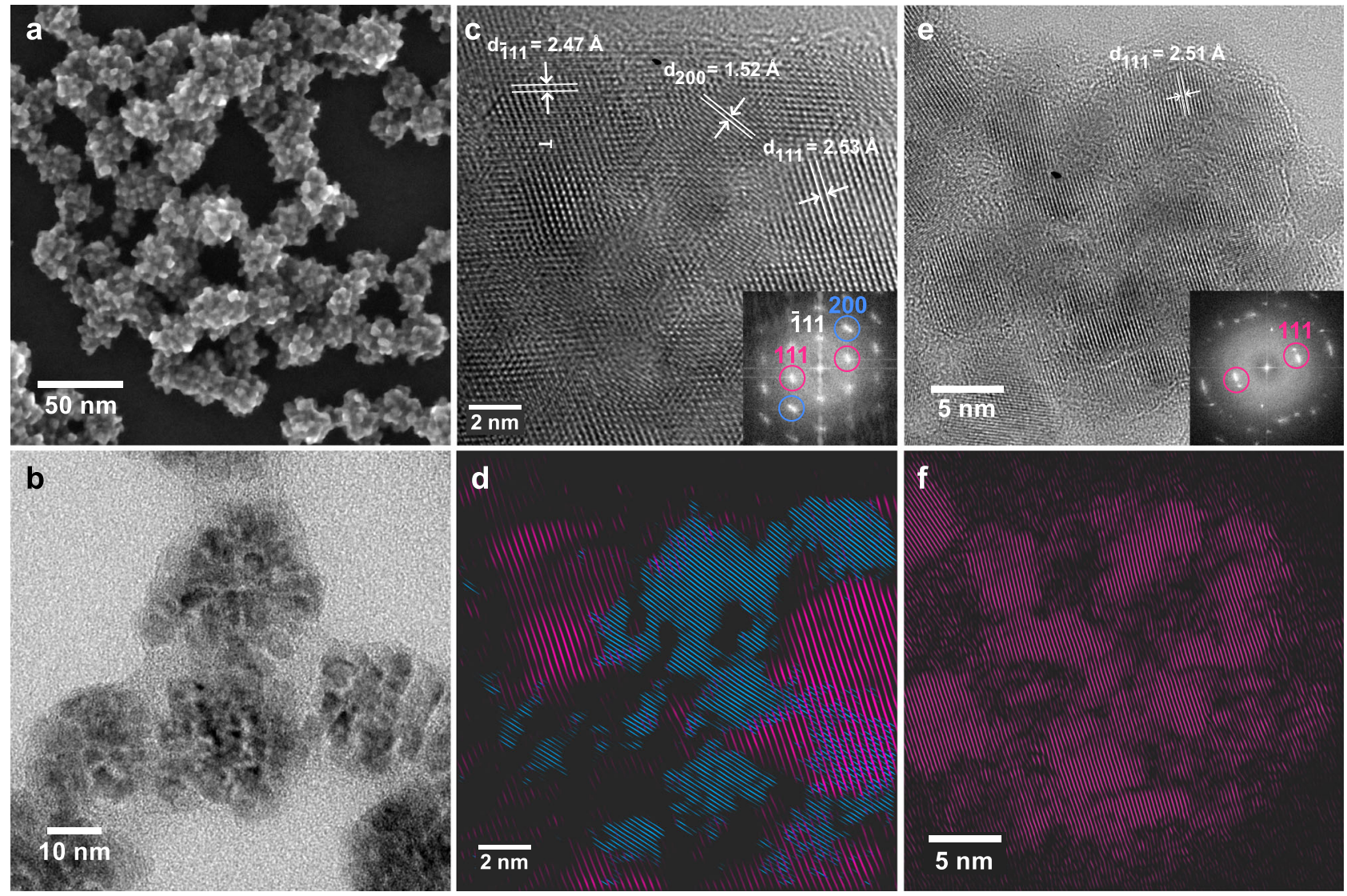

Fig. 1 SEM and TEM images of CoO assemblies at the end of the reaction. Different magnifications highlight their hierarchical structure. a Probecorrected SEM image of spherical assemblies built of irregularly shaped crystallites. $\mathbf{b}$ TEM image of assemblies grown after 90 min of reaction time. $\mathbf{c}$ HRTEM image and the corresponding Fourier transformation (FT) (inset) reveal that an assembly exhibits different crystallite orientations. A dislocation in the (111) plane is marked with a white T. d Filtered image of $\mathbf{c}$ by inverse FT of the frequency components highlighted by circles in c selectively showing the (111) orientation in violet and the (200) orientation in blue. e HR-TEM image of a different assembly and its corresponding FT, and $\mathbf{f}$ filtered inverse FT of e highlighting only the (111) orientation. Individual crystallites do not align to each other in a common crystallographic orientation. 
$\mathrm{CoO}$, but also to $\mathrm{Co}_{3} \mathrm{O}_{4}$, and to metallic $\mathrm{Co}$ particles. Interestingly, the crystal structure of individual $\mathrm{CoO}$ nanoparticles determines the growth mechanism. CoO nanoparticles with rocksalt structure have the tendency to form polycrystalline assemblies, while wurtzite $\mathrm{CoO}$ preferentially grows into single crystals $^{21,51,54}$. The specific mechanism in solution, however, favoring crystallization into one or the other phase, and the interdependence of crystallite growth and its assembly into superstructures, remains unsolved. Here, starting from $\mathrm{Co}$ (III) acetylacetonate (acac), we synthesize phase-pure $\mathrm{CoO}$ assemblies with an average size of $58 \mathrm{~nm}$ composed out of smaller, polyhedrally shaped, edgy 5-7-nm large particles. We show that HERFD-XANES enables us to track the rearrangement of the organometallic precursor complex accompanying the initial reduction of $\mathrm{Co}^{3+}$ to $\mathrm{Co}^{2+}$ in solution. PDF reveals the transition from the dissolved $\mathrm{Co}^{2+}$ complex to rock-salt $\mathrm{CoO}$ nanocrystals by monitoring the changes in bond length between the metal ion and the surrounding oxygen atoms. The combination of PDF and SAXS finally elucidates the interdependence of crystallite growth and assembly that results in the final morphology.

\section{Results}

The reaction of $\mathrm{Co}(\mathrm{acac})_{3}$ with benzyl alcohol $(\mathrm{BnOH})$ yields spherical $\mathrm{CoO}$ assemblies. They are composed of polyhedrally shaped, edgy nanocrystallites as shown in scanning electron microscopy (SEM) and high-resolution transmission electron microscopy (HR-TEM) images in Fig. 1. From TEM images recorded at different reaction times, we estimate that both the smaller crystallites and the assemblies grow with reaction time (Supplementary Fig. 2-4). Distinct particles are first visible in TEM after 20 min (Supplementary Fig. 5). The crystallites reach an average size of $6 \mathrm{~nm}$ and the assemblies have a final size between 20 and $45 \mathrm{~nm}$. The HR-TEM image of a single assembly in Fig. 1c reveals an arbitrary orientation of the crystallites, since different crystallographic planes are exhibited within the same assembly. This can be more easily seen in Fig. 1d and $f$ where we Fourier-filter the HR-TEM image of a single assembly, highlighting crystallites exhibiting the (111) planes in violet and those exhibiting the (200) planes in blue. The assembly of polyhedrally shaped particles into spherical structures implies a nonclassical multistep reaction pathway. To access the complexity of the reaction mechanism, we utilize complementary in situ X-ray spectroscopic and scattering methods.

Tracking chemical changes of the reactants via in situ X-ray absorption spectroscopy. To elucidate the chemical reactions in solution, we recorded in situ HERFD-XANES spectra of the $\mathrm{CoO}$ assembly synthesis at $160^{\circ} \mathrm{C}$. The full time series is shown in Supplementary Fig. 6 . There we notice that, immediately after the reaction temperature is reached, the absorption edge shifts by $3.4 \mathrm{eV}$ toward lower energies, indicating the reduction of the $\mathrm{Co}^{3+}$ precursor. Figure 2a depicts four representative stages of the time series in more detail. The multiple features in the preedge (second row in Fig. 2a) at 7709.5, 7712.5, and $7716 \mathrm{eV}$ that are present at the beginning of the reaction, reduce to a single feature at $7708.5 \mathrm{eV}$. These pre-edge features originate from hybridization of $3 \mathrm{~d}$ and $4 \mathrm{p}$ unoccupied states in $\mathrm{Co}^{2+}$ and $\mathrm{Co}^{3+}$, allowing quadrupole transitions of a 1 s core electron into unoccupied $3 \mathrm{~d}$ states ${ }^{55-57}$. Toward the end of the reaction, we observe an increase of the intensity of the white line at $7726 \mathrm{eV}$ that corresponds to $1 \mathrm{~s} \rightarrow 4 \mathrm{p}$ transitions in $\mathrm{CoO}$, confirming the formation of the final product ${ }^{56-58}$.

We use a multivariate curve resolution by alternating least squares (MCR-ALS) analysis to recover the number and spectra of independent compounds in the reaction mixture and follow changes of their concentration over time ${ }^{59,60}$. This way, we determine the presence of three independent compounds in the in situ data. Further details on the MCR-ALS analysis are given in Supplementary Notes 1 and Supplementary Fig. 7. By comparing linear combinations of the three recovered spectra with the in situ data at different reaction stages (Fig. 2a), we conclude that these compounds accurately describe both the overall spectral features, including the position of the absorption edge and the post-edge fluctuations as well as the pre-edge region. The residual portion of the experimental data not described by the three compounds is $2.1 \%$.

In Fig. 2b, we compare the recovered compound spectra to references. We find that the compound mostly present at the beginning of the reaction (shown in red) is in very good agreement with the reference spectrum of $\mathrm{Co}(\mathrm{acac})_{3}$ dissolved in benzyl alcohol. We determine the edge position at $7723.6 \mathrm{eV}$ (labeled B in Fig. 2b) confirming the valency of the precursor 64,65 . For the other compounds, the edge is observed at $7720.2 \mathrm{eV}$ (labeled $\mathrm{A}$ in Fig. 2b), indicating that both are in the oxidation state $\mathrm{Co}^{2+66-68}$. At the end of the reaction, the predominant compound (violet) reproduces all features of the $\mathrm{CoO}$ reference that was measured as a dry powder and corrected for selfabsorption $^{69}$ (for details, see Supplementary Notes 2 and Supplementary Fig. 8).

Most importantly, the compound shown in orange in Fig. 2 compares well to the $\mathrm{Co}(\mathrm{acac})_{2}$ reference, which we identify as a reaction intermediate. This is affirmed by the simulation of XANES spectra of $\mathrm{Co}(\mathrm{acac})_{2}$ using the FEFF code ${ }^{61}$ as shown in Fig. 3 and Supplementary Fig. 9, 10 (refer to the Supplementary Notes 4 and Supplementary Table 3 for details on the FEFF calculations). Furthermore, FEFF calculations allow deeper insight into the conformation of the reaction intermediate, since the spectral features are very sensitive to the local chemical environment around the absorbing $\mathrm{Co}^{2+}$ ion. Tetrahedral and square-planar conformations have been suggested for the isolated $\mathrm{Co}(\mathrm{acac})_{2}$ molecule ${ }^{62}$; however, only the calculated XANES spectra of an octahedrally coordinated $\mathrm{Co}^{2+}$ ion are in good agreement with the experiment. We thus conclude that in solution, the $\mathrm{Co}^{2+}$ ion is additionally coordinated by oxygen atoms of two solvent molecules, forming a bis-adduct of the square-planar $\mathrm{Co}(\mathrm{acac})_{2}$ with octahedral coordination ${ }^{70}$. Due to the limited solubility of $\mathrm{Co}(\mathrm{acac})_{2}$ in $\mathrm{BnOH}$ at room temperature, the reference for the MCR-ALS analysis was measured as a powder where the complex is known to form the tetramer $\mathrm{Co}_{4}(\mathrm{acac})_{8}$ that also exhibits octahedral coordination ${ }^{70,71}$ (Supplementary Fig. 11d). Thus, we attribute the deviations between the spectrum of the reaction intermediate recovered by MCR-ALS and the $\mathrm{Co}(\mathrm{acac})_{2}$ reference in Fig. $2 \mathrm{~b}$ to slight changes in the coordination geometry and the second-shell chemical environment upon dissolving.

To quantify the portion of each compound in the system, in Fig. 2c, we examine the profiles of the relative concentrations over time that result from the MCR-ALS analysis. When the temperature reaches the set point, $\mathrm{BnOH}$ reduces $\mathrm{Co}(\mathrm{acac})_{3}$ to $\mathrm{Co}(\mathrm{acac})_{2}$ within $10 \mathrm{~min}$, significantly faster than the formation of $\mathrm{CoO}$ from $\mathrm{Co}(\mathrm{acac})_{2}$. Both reaction steps are best described by pseudo-first-order kinetics ${ }^{72,73}$ (for details, see Supplementary Notes 3 and Supplementary Fig. 9) with the rate of the initial reduction being significantly higher than that of the formation of the final product. We ascribe the fluctuations in the relative concentrations most notable in the beginning and at the end of the reaction to changes of the position of the X-ray beam on the reaction cell, in combination with no stirring applied. This can be a reason why the relative concentration of $\mathrm{Co}(\mathrm{acac})_{2}$ never reaches $100 \%$. Precipitation of large particles could be another reason why this is neither the case for CoO. However, it is 

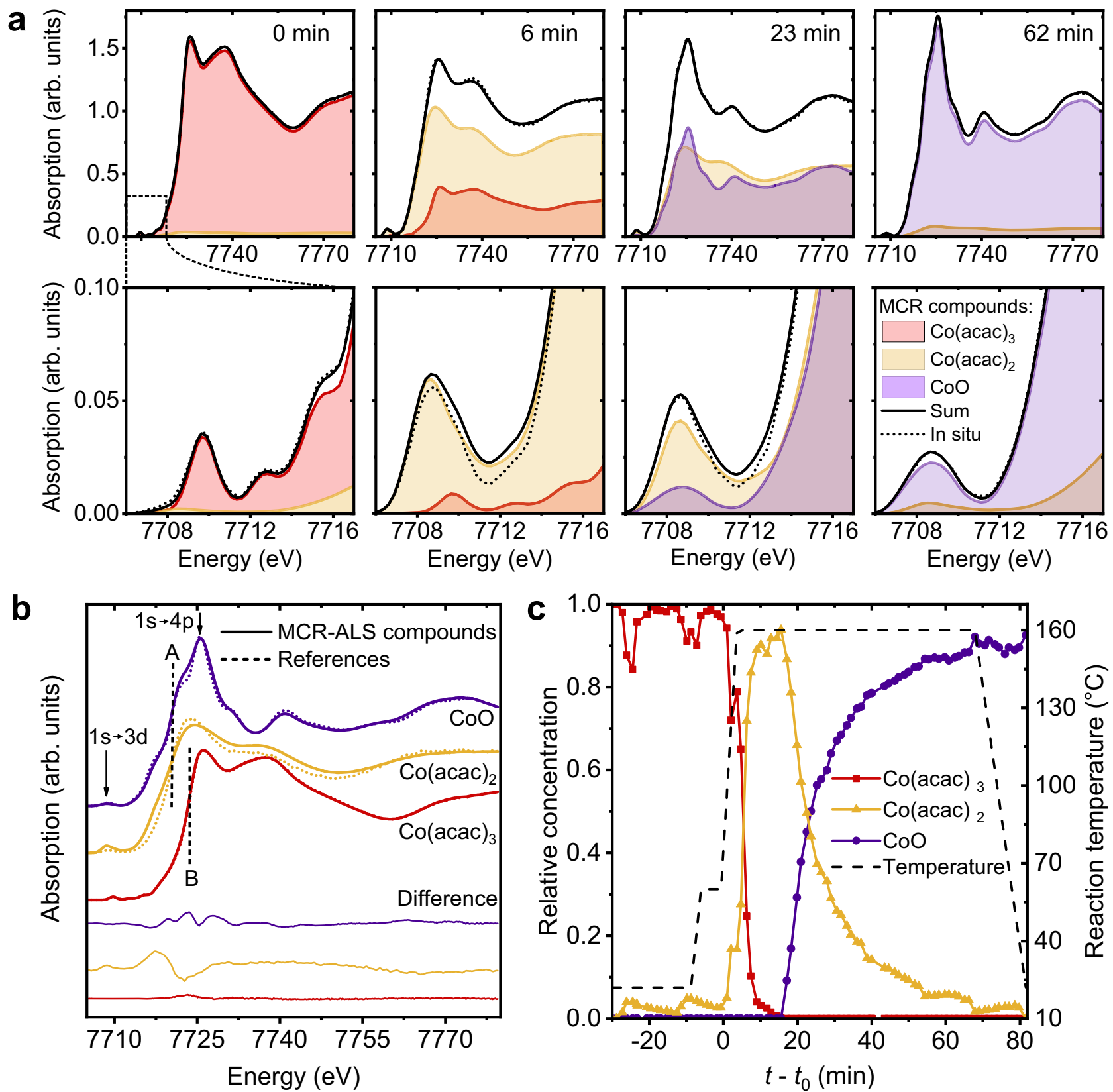

Fig. 2 Determination of chemical constituents from the in situ HERFD-XANES study and the MCR-ALS analysis. a Selected HERFD-XANES spectra of the time series with the main edge shown in the first and the pre-edge in the second row. The spectra are plotted together with the multivariate curve resolution by alternating least squares (MCR-ALS) compounds recovered at the respective times. Linear combinations of the recovered spectra (black solid lines) match the in situ data (dotted lines). b Spectra of the recovered MCR-ALS compounds compared with measured reference spectra of Co(III) acetylacetonate (acac) dissolved in benzyl alcohol (red), $\mathrm{Co}(\mathrm{acac})_{2}$ (orange), and $\mathrm{CoO}$ (violet). Dashed vertical lines emphasize the edge positions of $\mathrm{Co} 2+$ (" $\mathrm{A}$ " at $7720.2 \mathrm{eV}$ ) and $\mathrm{Co}^{3+}$ ("B" at $7723.6 \mathrm{eV}$ ). c Relative concentrations of the MCR-ALS compounds over reaction time. The dashed line indicates the temperature set point. We set $t_{0}$ (beginning of reaction time) to the point when we start heating to $160^{\circ} \mathrm{C}$.

important to note that the formation of $\mathrm{CoO}$ only sets in after the precursor has been completely reduced to $\mathrm{Co}(\mathrm{acac})_{2}$. We can thus exclude the possibility of parallel reactions such as the precursor reacting to $\mathrm{CoO}$ without a stable intermediate.

Pinning down nucleation and growth of nanoparticles with in situ PDF. X-ray spectroscopy provides information about the chemical state of cobalt species. However, it fails to investigate the crystallization and growth of the $\mathrm{CoO}$ nanoparticles from a structural perspective. Therefore, we performed in situ total X-ray scattering experiments of the synthesis at $160^{\circ} \mathrm{C}$. Supplementary Fig. 12 shows the integrated and background-subtracted in situ total X-ray scattering data. The constant broad feature at around $1 \AA^{-1}$ results from the remaining scattering from the sample environment (glass vessel filled with $\mathrm{BnOH}$ ) after background subtraction (Supplementary Fig. 13). Except for the residual background, no reflections are seen either in the initial stages of the reaction or during the step of heating. The crystallization of $\mathrm{CoO}$ starts after ca. 20 min of reaction time suggested by the appearance of $\mathrm{CoO}$ reflections, which persist until the reaction finishes after $90 \mathrm{~min}$. 

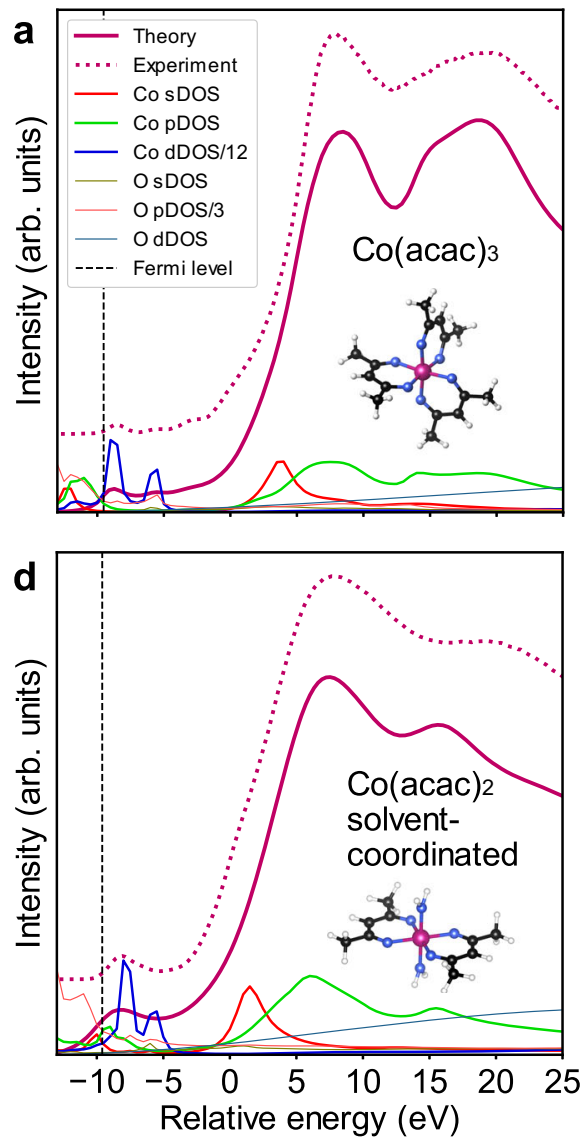
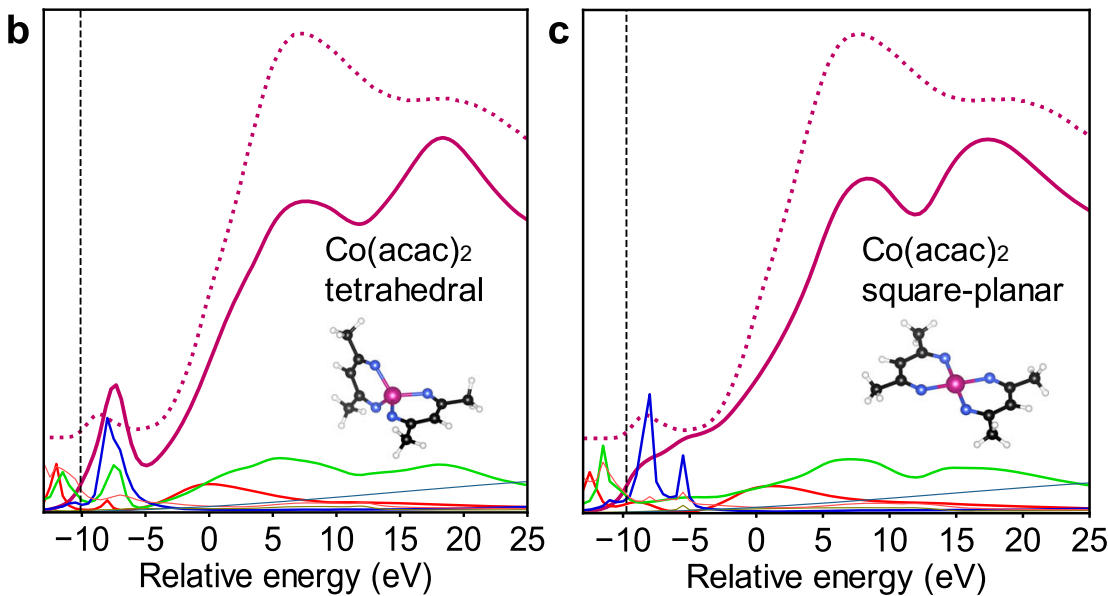

e
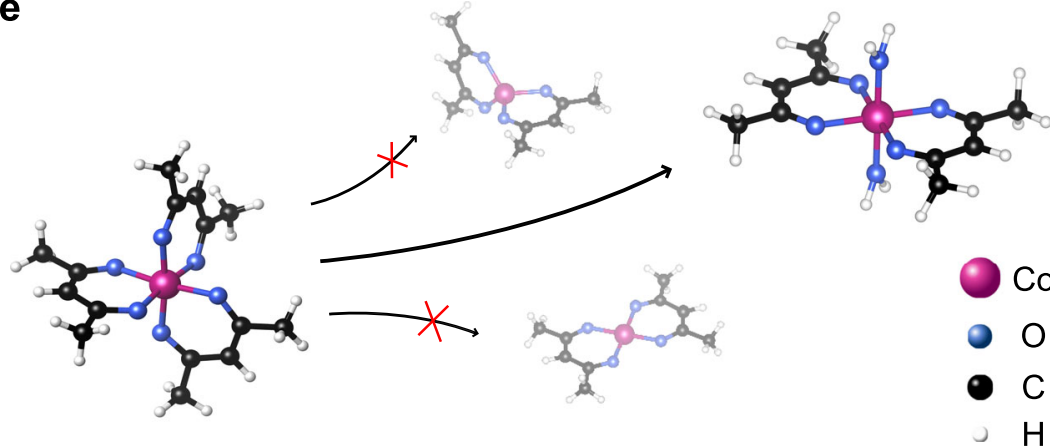

Fig. 3 Structure determination of the reaction intermediate Co(acac) $)_{2}$. The structure is determined by means of theoretical XANES spectra calculated using the FEFF code ${ }^{61}$. The spectrum of $\mathrm{Co}(\mathrm{III})$ acetylacetonate (acac) is shown in a for comparison. Examined structures include $\mathbf{b}$ tetrahedral, $\mathbf{c}$ squareplanar and $\mathbf{d}$ solvent-coordinated with water as a model solvent, since the precise coordination geometry of benzyl alcohol is unknown. The recovered spectra from the MCR-ALS analysis corresponding to $\mathrm{Co}(\mathrm{acac})_{3}$ and $\mathrm{Co}(\mathrm{acac})_{2}$ are shown as dotted lines for reference in parts $\mathbf{a}$ and $\mathbf{b}$-d, respectively. Spectra are plotted together with the local density of states (DOS) for $s, p$ and $d$ states of cobalt and oxygen atoms. The energy scale is relative to the absorption edge $E_{0}$. The position of the Fermi level is indicated for each structure as a vertical dashed line. No instrumental broadening was necessary for the energy resolution of FEFF calculations to be in good agreement with the experimental HERFD-XANES spectra (for details, see Supplementary Notes 4). e Structural models of the proposed reaction pathway. The atomic positions in square-planar and tetrahedral $\mathrm{Co}(\mathrm{acac})_{2}$ were taken from reference ${ }^{62}$ and the structure of $\mathrm{Co}(\mathrm{acac})_{3}$ was adopted from reference ${ }^{63}$.

Total scattering data encode structural information on chemical constituents independent of their aggregation state and ordering, and thus enable to study precursor molecules in solution, as well as amorphous and crystalline nanoparticles. To access this information, we convert the in situ-recorded scattering data into the time-resolved PDFs $(\mathrm{G}(r))$, as shown in Fig. 4a. We obtain a real-space representation of the interatomic distance changes of all compounds in the reaction mixture over the course of the reaction. At the beginning, the time-resolved PDFs reveal only a short-range order within a correlation length $r$ of less than $5 \AA$, indicating the presence of isolated molecular species. The long-range order suddenly appears after $20 \mathrm{~min}$, pinning down the nucleation of the crystalline $\mathrm{CoO}$ phase.

The low $r$ region of the time-resolved PDFs before the crystallization as seen in Fig. $4 \mathrm{~b}$ clearly shows a single and intense feature at $r$ ca. $2 \AA$ that is constant until longer-range order appears. This peak represents the nearest-neighbor $\mathrm{Co}-\mathrm{O}$ bond. However, the position of this peak starts shifting to higher $r$ values only $4 \mathrm{~min}$ after heating to the final reaction temperature is started, revealing the initial changes of the Co environment in the precursor prior to nucleation. To quantify this behavior, the PDFs corresponding to the selected reaction stages are depicted in Fig. 4c. At early reaction times below $13 \mathrm{~min}$, the PDFs show only local order of the reactant. As the reaction progresses further to
$20 \mathrm{~min}$ and beyond, the peaks related to the structure of $\mathrm{CoO}$ first on the short-to-medium range and soon also on the long-range emerge and intensify as indicated by the dashed lines. Moreover, small features at around 2.34, 2.76, and $3.14 \AA$ in the initial stage match very well with the $\mathrm{C}-\mathrm{O}$ and two different $\mathrm{Co}-\mathrm{C}$ bond distances in the $\mathrm{Co}(\mathrm{acac})_{3}$ molecule $^{63}$. These peaks change position over time and disappear when the crystalline $\mathrm{CoO}$ phase appears, most noticeably when the peak of the nearest Co-Co distance at around $3.01 \AA$ emerges. The overall behavior is similar to ex situ reference measurements after selected reaction times, as shown in Supplementary Fig. 14a,b.

The observed shifts of PDF peaks during the reaction suggest that the effective $\mathrm{Co}-\mathrm{O}$ distance can act as a fingerprint of the nucleation pathway. To test this approach, we depict the changes in the shortest $\mathrm{Co}-\mathrm{O}$ distance over reaction time in Fig. $4 \mathrm{~d}$. Simple eye inspection reveals three stages for the reaction. At room temperature and during heating, the $\mathrm{Co}-\mathrm{O}$ distance remains close to $\sim 1.90 \AA$ (stage 1), which agrees well with the bond length obtained from our ex situ measurements of the $\mathrm{Co}(\mathrm{acac})_{3}$ reference (Supplementary Fig. 14c). After about $4 \mathrm{~min}$, the $\mathrm{Co}-\mathrm{O}$ bond length rapidly increases and fluctuates around $1.98 \AA$ until $20 \mathrm{~min}$, suggesting an intermediate state (stage 2). Then, the Co-O distance gradually expands further up to $30 \mathrm{~min}$, where it approaches a value of $\sim 2.12 \AA$ that remains close to 

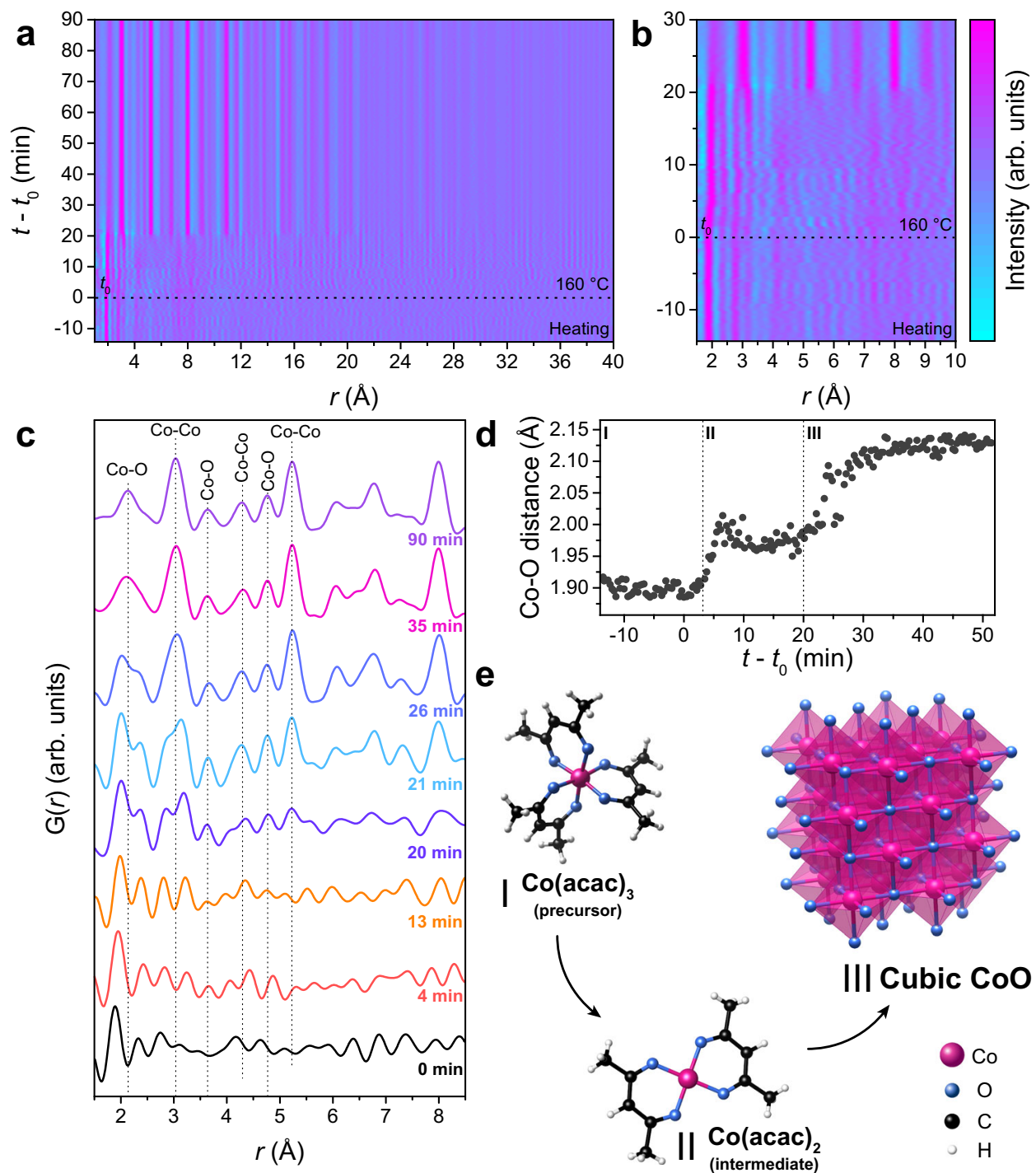

Fig. 4 Following the structural rearrangement of the Co complex and the crystallization of CoO. a In situ time-resolved PDFs G( $r)$ for the CoO synthesis. The heating step includes heating from room temperature to $60^{\circ} \mathrm{C}, 5 \mathrm{~min}$ at $60^{\circ} \mathrm{C}$, and heating from 60 to $160^{\circ} \mathrm{C}$. We set $t_{0}$ (beginning of reaction time) to the point when we start heating to $160^{\circ} \mathrm{C}$. b Region of $\mathbf{a}$ illustrating the short-range order before the crystallization of CoO. $\mathbf{c}$ Zoom into the local order region of the PDFs for selected reaction times of the in situ data. Dashed lines show the first coordination shells of CoO. $\mathbf{d}$ Changes in the shortest $\mathrm{Co}-\mathrm{O}$ bond over the reaction course, which indicate three reaction stages. e Structural models for the proposed reaction stages. The structures were obtained from the crystallographic data from references $63,74,75$ using the VESTA software ${ }^{76}$.

constant (stage 3 ). It is worth noting that the transformation from stage 1 to 2 occurs much faster than the final changes from stage 2 to 3, matching the reaction rates found by the HERFD-XANES study (Fig. 2c).

The reaction stages can thus be explained as follows. Stage 1 is associated with the precursor $\mathrm{Co}(\mathrm{acac})_{3}$ without noticeable changes. For stage 2, HERFD-XANES pointed out $\mathrm{Co}(\mathrm{acac})_{2}$ as the reaction intermediate at comparable reaction times as those inferred from Fig. 4d. Thus, it is reasonable that the Co-O bond lengthening associated with the lack of long-range order corresponds to the reduction of the precursor to $\mathrm{Co}(\mathrm{acac})_{2}$ during stage 2, although the ex situ measurement of the $\mathrm{Co}(\mathrm{acac})_{2}$ reference at room temperature provides a slightly longer $\mathrm{Co}-\mathrm{O}$ bond length of $2.05 \AA$ (Supplementary Fig. 14c). Finally, stage 3 corresponds to the formation of $\mathrm{CoO}$ nanoparticles, as the $\mathrm{Co}-\mathrm{O}$ distance approaches that of the first coordination shell in rocksalt $\mathrm{CoO}$. The proposed reaction course is illustrated in Fig. 4e.

To track the structural evolution of the $\mathrm{CoO}$ particles in the final phase 3 , we perform a sequential refinement on the time- resolved PDFs starting from the final product after $90 \mathrm{~min}$, and trace the signal back toward earlier reaction times. In this process, calculated PDFs are fitted to the data in the $r$ range between 1.5 and $40 \AA$ applying the crystal structure of cubic CoO. Besides other structural parameters, we refine the spherical particle (sp) diameter that represents the size of individual crystallites in PDF. Figure $5 \mathrm{a}$ shows the sp diameter as a function of the reaction time. The refinement results for the other parameters are depicted in Supplementary Fig. 15. At $21 \mathrm{~min} 20 \mathrm{~s}$, the initial value of the sp-diameter is $\sim 35 \AA$, increasing rapidly to ca. $50 \AA$ within additional $10 \mathrm{~min}$. Crystallites gradually continue to grow until the end of the reaction at $90 \mathrm{~min}$ and reach a diameter of $64 \AA$. At the final reaction stage, the crystallite sizes are in good agreement with the size of $6 \mathrm{~nm}$ of the small building blocks of the assemblies visible in TEM images as shown in Fig. 1. The lattice parameter $a$ rapidly decreases as the reaction progresses and fluctuates around $4.28 \AA$ throughout most of the reaction time. It is worth noting that the sequential refinement was also carried out in a higher $r$ range of $1.5-60 \AA$, comparable to the size of the 

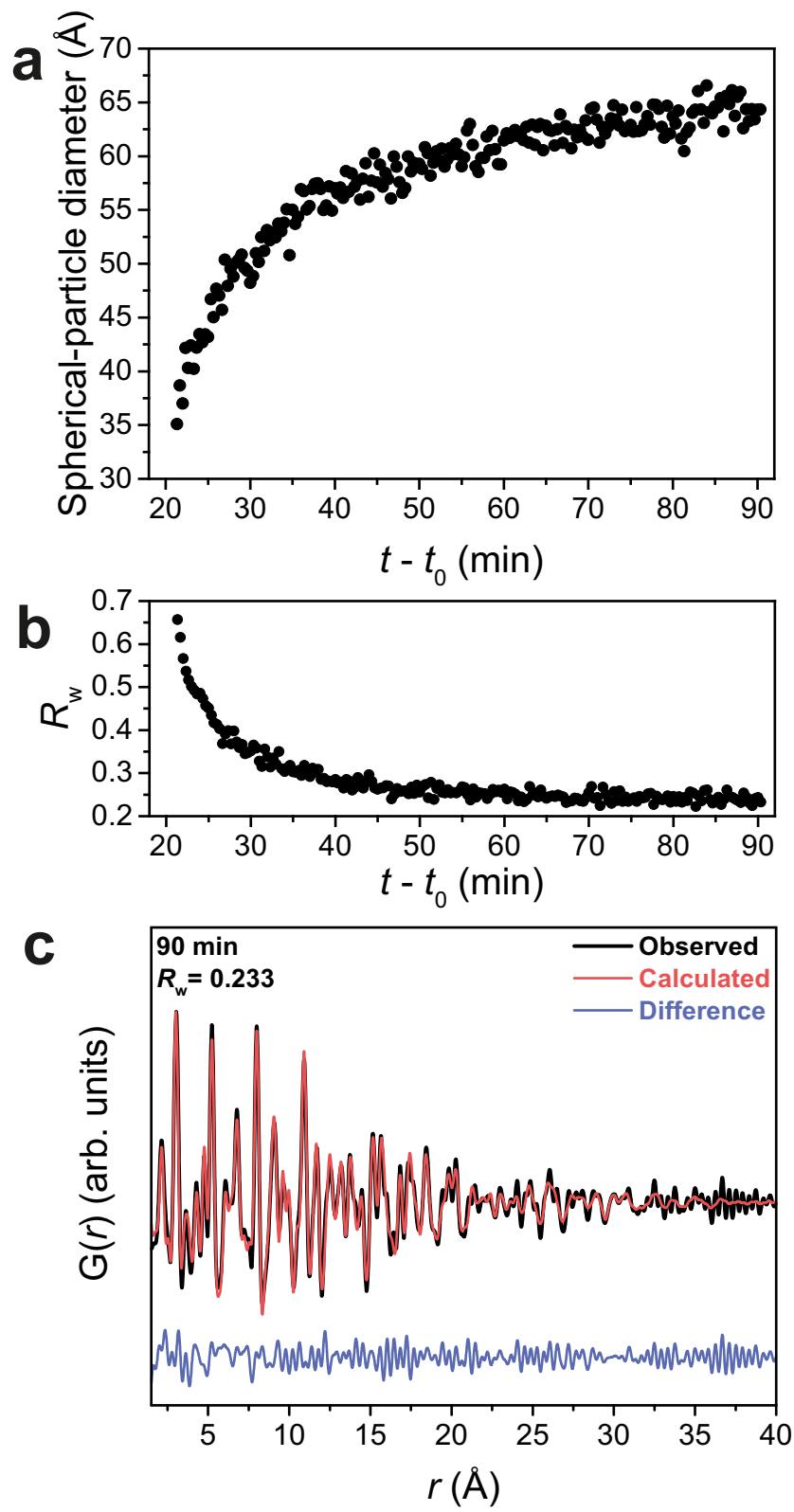

Fig. 5 Results of the sequential PDF refinement. a Evolution of $s p$ diameter as a function of the reaction time. $\mathbf{b}$ Fit-quality $R_{\mathrm{w}}$ progression as a function of the reaction time. The data in $\mathbf{a}$ and $\mathbf{b}$ were obtained from the sequential refinement of time-resolved PDFs. $\mathbf{c}$ Experimental and calculated PDFs $\mathrm{G}(r)$ for the reaction at $160^{\circ} \mathrm{C}$ after $90 \mathrm{~min}$. The calculation imposes a rock-salt $\mathrm{CoO}$ phase ${ }^{75}$.

small building blocks, and similar refinement results are observed as illustrated in Supplementary Fig. 16.

The fit-quality $R_{\mathrm{w}}$ (lower is better) corresponding to the PDF refinement presented in Fig. 5a and Supplementary Figure 15 is shown in Fig. $5 b$ as a function of the reaction time. For the reaction time before $40 \mathrm{~min}$, the co-existence of $\mathrm{Co}(\mathrm{acac})_{2}$ and $\mathrm{CoO}$ induces a misfit in the low $r$ region. The sp diameter is robust against the misfit in this region and thus the relative changes of crystallite size are reliable. However, the $\mathrm{CoO}$ structural model does not well describe the experimental data before $40 \mathrm{~min}$ as shown in Supplementary Fig. 17a. After $40 \mathrm{~min}$ and beyond, the PDF data and fit match well, with $R_{\mathrm{w}}$ dropping below 0.3 , and improving continuously with reaction time. The modeling of the PDF obtained at $90 \mathrm{~min}$ reveals good agreement with the cubic $\mathrm{CoO}$ structure in the whole $r$ range, i.e., a low $R_{\mathrm{w}}$ of 0.233 , as depicted in Fig. 5c. Additional refinements of the PDFs at other reaction stages are shown in Supplementary Fig. 17b-d.

The in situ reaction at a milder temperature of $140^{\circ} \mathrm{C}$ compared with the reaction at $160{ }^{\circ} \mathrm{C}$ discussed so far presents a similar trend of the formation of the $\mathrm{CoO}$ nanoparticles. The kinetics and growth rate are, however, significantly slower than those at $160^{\circ} \mathrm{C}$, as discussed in Supplementary Notes 5 and Supplementary Figs. 18, 19.

Tracking the assembly with SAXS. So far, we have elucidated the reaction pathway from the dissolved precursor to the product phase. While with PDF analysis we have been able to follow nucleation and growth, information about the process of crystallite assembly into the superstructure is missing. The size and the internal structure of assemblies in solution are accessible from the scattered X-ray intensity at small scattering angles $2 \theta$, here below $4.2^{\circ}$. We show this SAXS data as a function of scattering vector $q=\frac{4 \pi}{\lambda} \sin (\theta)$, where $\lambda$ is the X-ray wavelength. SAXS intensities $I(q)$, shown in Fig. 6a, were recorded ex situ from aliquots obtained by ceasing the reaction at five different time points. After $10 \mathrm{~min}$ of reaction time, the SAXS intensity shows a pronounced divergence at low $q$. The intensity obeys approximately a $q^{-4}$ power law characteristic for scattering contrast from objects with well-defined boundaries ${ }^{77,78}$. The slight kink below $q=0.01 \AA^{-1}$ indicates that the characteristic length scale of the scattering objects $(2 \pi / q)$ is a few $10 \mathrm{~nm}$. At this reaction stage, the intermediate $\mathrm{Co}(\mathrm{acac})_{2}$ dominates the reaction mixture according to our HERFD-XANES measurements reported in Fig. 2c. Since the intermediate is not well soluble in $\mathrm{BnOH}$ at room temperature, the scattering signal visible in SAXS at this stage may be from precipitates of the intermediate.

From 20 min reaction time onward, the SAXS signal at low angles, i.e., low values of $q$, is well described by an intensity plateau region and a subsequent intensity drop. This is clear evidence that well-defined objects have formed. A fit with a model assuming homogeneous spherical assemblies (solid line in Fig. 6a, see also Supplementary Notes 6 and Supplementary Table 4) yields an assembly diameter of $D=21 \mathrm{~nm}$. Additionally, a size polydispersity of $\triangle D / D=25 \%$ is obtained. For 40,60 , and $90 \mathrm{~min}$ reaction time, the assembly diameter gradually increases, i.e., the plateau region narrows. The sphere size obtained from the analysis levels off at a diameter of $D=58 \mathrm{~nm}, \Delta D / D=19 \%$, as summarized in Fig. 6b.

For larger diameters, a second intensity oscillation is observed in the data close to the plateau region, as expected for scattering from homogeneous spheres. The homogeneous spherical model, however, only describes the SAXS intensity well up to $q=0.04 \AA^{-1}$. For larger $q$ values, the model strongly underestimates the SAXS intensity, indicating a more complex internal structure.

Particles with pronounced internal structure, such as fractal and porous materials, are known to show a crossover of the power law underlying the SAXS intensity, at the point where $q$ is large enough to resolve these internal structures ${ }^{77}$. Our data suggest that such a crossover from initially $q^{-4}$ to $q^{-2}$ occurs here at ca. $q=0.06 \AA^{-1}$, i.e., for length scales smaller than $10 \mathrm{~nm}$, illustrated by the dotted lines in Fig. 6a. The region described by $q^{-2}$ extends up to $q=0.2 \AA^{-1}$ (indicated by vertical lines) beyond which the $q^{-4}$ behavior is recovered. Thus, the scattering signal in the intermediate region originates from inhomogeneities at length scales of ca. $3 \mathrm{~nm}$ up to $10 \mathrm{~nm}$. This size range is considerably smaller than the full diameter of the assemblies but coincides with the size range of the building units determined with PDF (sp diameter in Fig. 5a). On an even smaller scale, i.e., for $q>0.2 \AA^{-1}$, the assemblies scatter like compact objects. In SAXS, the dimensional crossover is seen for all 

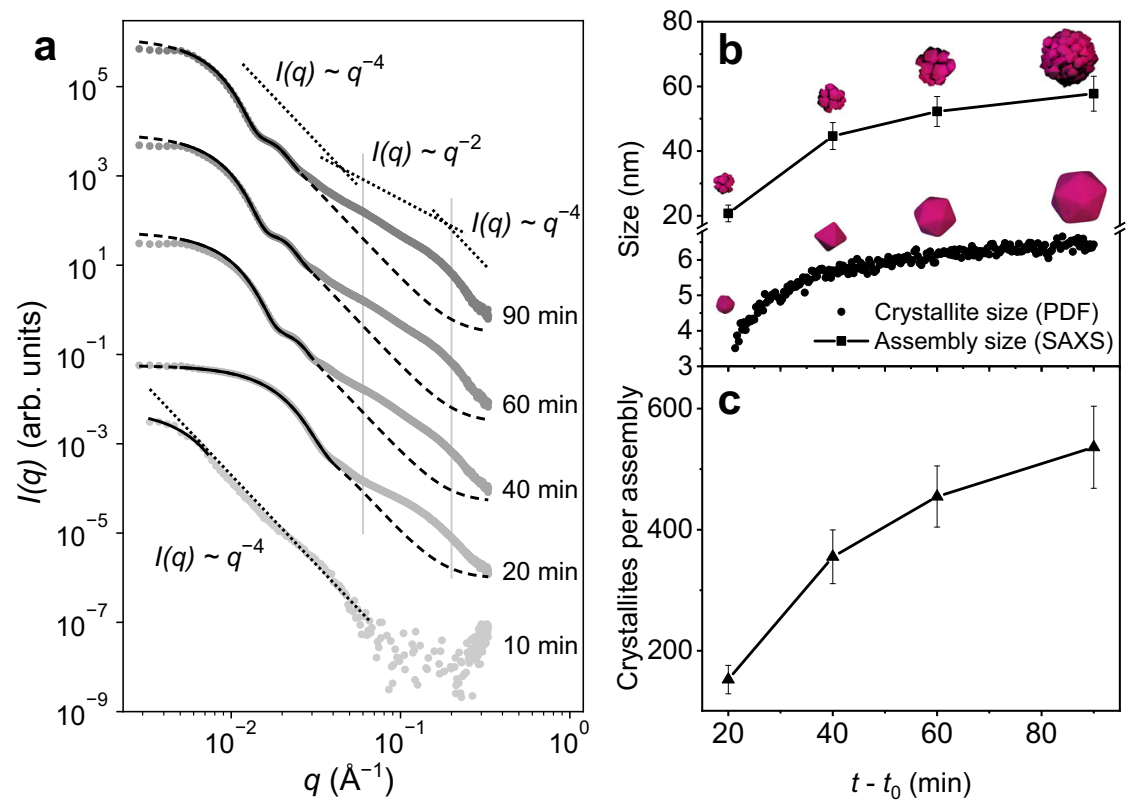

Fig. 6 SAXS data modeling to follow the assembly of CoO nanocrystals. a Small-angle X-ray scattering (SAXS) intensities I( $q$ ) of CoO assemblies for different reaction times. Dashed curves: calculated intensities for homogeneous spherical assemblies. Superimposed bold curves indicate the least-squares fitting range. Dotted lines: power laws describing the contributions of compact particle shape $\left(q^{-4}\right)$ and corrugated surface $\left(q^{-2}\right)$ on the different length scales. An intermediate region is emphasized by vertical gray lines. b Comparison of size evolution over time of polyhedra (spherical particle diameter obtained from in situ pair distribution functions (PDF)) and assemblies (obtained from SAXS). Here, the error bars indicate the polydispersity of the assemblies. c Average number of crystallites per assembly obtained from average assembly size in $\mathbf{b}$ assuming that they are built from densely packed spherical units with a filled volume fraction of $74 \%$. Here, the error bars result from the error propagation of the average assembly sizes (SAXS) and of the standard deviation of the crystallite sizes (PDF) in $\mathbf{b}$.

reaction times between 20 and 90 min, suggesting that the assemblies are corrugated and porous throughout the reaction. The SEM and TEM images shown in Fig. 1 and Supplementary Figs. 2, 3 and 22 also display that the compact crystallites form porous assemblies with voids in the interior.

We measured samples after selected reaction times in our laboratory SAXS system. Here, we are able to calibrate the SAXS data on absolute scale, allowing us to determine the volume fraction of the reactant (for details, see Supplementary Notes 6). At the end of the reaction, the volume fraction of the assembly is $0.026 \%$, corresponding to a $\mathrm{CoO}$ concentration of $1.65 \mathrm{mg} / \mathrm{ml}$ and a reaction yield of ca. $22 \%$. However, since these samples were measured ex situ, precipitation may have led to an underestimation of the concentration and thus the reaction yield. The final assembly size of $D=58 \mathrm{~nm}$ reported by SAXS is in good agreement with TEM images taken from the same batch used for SAXS analysis (Supplementary Fig. 22). However, assemblies are about 30\% larger than those from another batch shown in TEM images of Fig. 1. Even though the absolute values vary between the batches, we observed the similar growth trends independent of the batch. We measured PXRD for the same samples as used for the SAXS analysis, yielding cubic CoO domain sizes of $4.9 \pm$ $2.8 \mathrm{~nm}, 5.8 \pm 1.0 \mathrm{~nm}$, and $6.0 \pm 1.2 \mathrm{~nm}$ by Scherrer analysis for 40, 60 , and $90 \mathrm{~min}$ reaction times, respectively. For details, refer to Supplementary Table 5 and Supplementary Fig. 21. These values are in good agreement with the crystallite sizes found by the in situ PDF analysis.

The average number of crystallites per assembly can now be estimated as a function of reaction time. For this purpose, we compare the assembly size as obtained from SAXS with the diameter of the crystallites, i.e., the sp diameter as obtained from the PDF refinement (Fig. 6b). We plot the resulting number of crystallites per assembly in Fig. $6 c$, assuming dense packing of spherical units with a filled volume fraction of $74 \%$. From the similarity of the three curves, we conclude that during the entire evolution of the $\mathrm{CoO}$ phase, both the attachment of crystallites onto the assemblies and the growth of the individual crystallites contribute similarly to the resulting growth of the assemblies. Moreover, time-resolved TEM images shown in Supplementary Fig. 2 and 3 support our findings and show that during all reaction stages, crystallites in the center of the assemblies are of the same size as the ones at the surface.

\section{Discussion}

Previous studies of nonclassical crystallization mechanisms suggest that multiple pathways exist involving distinct precrystalline entities (see Cölfen et al. $2019^{10}$, Fig. 1d therein and references). Our work provides an experimental complement to this work. Using the example of polycrystalline assemblies of polyhedrally shaped $\mathrm{CoO}$ nanoparticles, we illustrate that advanced X-ray spectroscopic and scattering in situ studies are very powerful tools for bridging the molecular- and macro length scales. Putting together complementary pieces of information, we can pin down the formation of monomers, nucleation, growth, and assembly of the CoO polyhedra as schematically shown in Fig. 7 and Supplementary Fig. 23. In summary, HERFD-XANES revealed $\mathrm{Co}(\mathrm{acac})_{2}$ as a monomer species that quickly forms upon reduction of the precursor in solution. FEFF calculations indicated that the intermediate forms a bis-adduct of square-planar $\mathrm{Co}(\mathrm{acac})_{2}$ with coordinated solvent molecules. Total scattering and PDF analysis gave insights into local structural changes around the $\mathrm{Co}^{2+}$ ion during nucleation and growth of the cubic $\mathrm{CoO}$ phase. We assigned the stepwise increase of the $\mathrm{Co}-\mathrm{O}$ bond length to the rearrangement of the organo-metallic precursor upon reduction, and to the nucleation of the crystalline product phase. SAXS measurements together with crystallite-size evolution derived from PDF shed light on the assembly mechanism of the final structure. They indicate that crystallites start assembling 


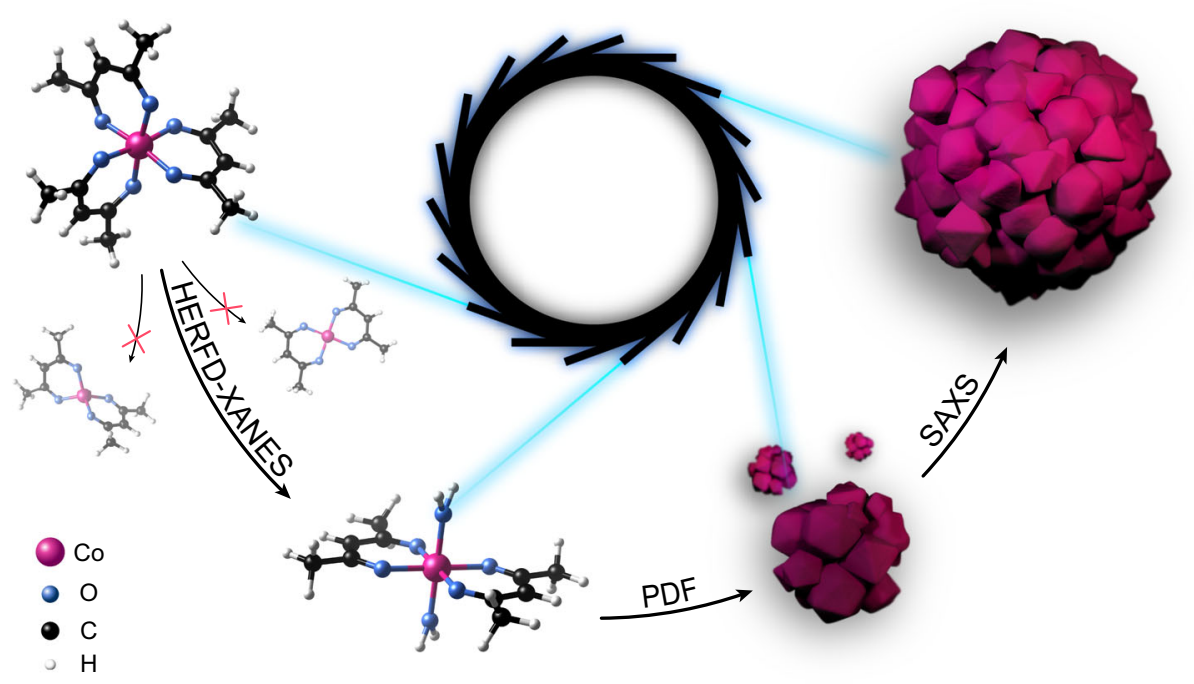

Fig. 7 Overview of the emergence of nano-assemblies of $\mathbf{C o O}$ in benzyl alcohol. Complementary X-ray spectroscopic and scattering methods enable the bridging of the studies of the reaction mechanism from molecular- to macro- length scales. We combined high energy-resolution fluorescence-detected $X$ ray absorption near edge structure (HERFD-XANES), pair distribution function (PDF) analysis and small-angle X-ray scattering (SAXS) to follow the emergence of $\mathrm{CoO}$ nanoassemblies in solution.

soon after nucleation and continue to assemble, while the cavernous nature of the assemblies allows crystallites to grow similarly at the surface and in bulk. During the entire reaction, growth and assembly of particles contribute to the size evolution of the final product. The size evolution of crystallites and assemblies, as well as the average number of crystallites per assembly, follows the kinetics of the formation of the product phase. Our findings do not support a classical crystallization and growth mechanism, in which crystallites nucleate and grow only at the surface of the assemblies, until they are covered with the next layer of crystallites. On the contrary, the cavernous structure of the nanoparticle assemblies allows the crystallites to always consume material from the solution and thus to grow both on the surface and in bulk. In addition, no isolated, unassembled crystallites are found in SAXS or TEM, indicating that crystallites, which may nucleate in solution, are soon attached to an assembly, keeping the number of isolated crystallites low.

The simultaneous growth and assembly of crystallites we observe here is a markedly different mechanism from, e.g., evaporation-induced assembly of pre-formed nanocrystals in dispersion to mesocrystals, which has been in the focus of recent in situ investigations by X-ray methods ${ }^{79-81}$. Here we find that for the synthesis of $\mathrm{CoO}$ nanocrystals under solvothermal conditions, crystallite nucleation and growth are accompanied by unoriented assembly into a polycrystalline superstructure.

More generally, our approach of combining three techniques from the fields of X-ray spectroscopy and scattering made it possible to gain mechanistic insights into all steps of complex nonclassical crystallization. While spectroscopy is focused on the chemistry, scattering in the full range of reciprocal space is essential to follow the structural and morphological evolution at different length scales, both key features when it comes to understanding the complexity of nanomaterial formation.

We demonstrate a way to strengthen the use of multimodal in situ X-ray experiments, in particular, for studying nonclassical crystallization processes. The level of microscopic detail obtained within this study encourages the application of this approach in related fields, which might benefit even more from the combination of structural and spectroscopic synchrotron techniques. Particularly, catalysts at functional interfaces, which are notoriously difficult to probe in a coherent chemical and structural way, might be worthwhile to explore ${ }^{82}$.

\section{Methods}

Synthetic procedure. Chemicals: Benzyl alcohol $(>99 \%)$, Co(acac) $3(99.99 \%)$, and $\mathrm{Co}(\mathrm{acac})_{2}(>99 \%)$ were purchased from Sigma-Aldrich, and ethanol (absolute) for washing from VWR. All chemicals were used without further purification.

Synthesis: $\mathrm{Co}(\mathrm{acac})_{3}(0.5 \mathrm{mmol})$ was added to $5 \mathrm{~mL}$ of $\mathrm{BnOH}$ and stirred for $5 \mathrm{~min}$ at room temperature. A quantity of $0.8 \mathrm{~mL}$ of the green solution was transferred to the reaction container of the in situ cell (Supplementary Fig. 1), sealed, and heated at an average heating rate of $1^{\circ} \mathrm{C} / \mathrm{s}$ first to $60^{\circ} \mathrm{C}$ for $5 \mathrm{~min}$ to homogenize, then at the same rate to $140^{\circ} \mathrm{C}$ or $160^{\circ} \mathrm{C}$, and stabilized at this temperature during $210 \mathrm{~min}$ or $90 \mathrm{~min}$, respectively, for in situ experiments. We define the beginning of the reaction time $\left(t_{0}\right)$ at the point when the heating to the reaction temperature of $140^{\circ} \mathrm{C}$ or $160^{\circ} \mathrm{C}$ starts. All mentions of reaction time are relative to $t_{0}$. For ex situ SAXS measurements, the reaction was stopped after 20, 40, 60 , and 90 min of reaction time by quickly cooling down the in situ cell to room temperature with a cold metal block, and the solution was measured without further treatment. For SEM/TEM, the brown precipitate was washed three times with ethanol and dried at $60^{\circ} \mathrm{C}$. For the TEM image in Supplementary Fig. 22, no washing was applied.

SEM: Scanning electron microscopy images were taken and probe-corrected with a Regulus 8220 (Hitachi High Technologies Corp., Japan) at an acceleration voltage of $10 \mathrm{kV}$ and using the secondary electron signal.

TEM: Transmission electron microscopy images and electron diffraction (ED) measurements were taken with a JEM 1011, high-resolution TEM images with a JEM 2200 FS (JEOL Ltd., Japan) at an acceleration voltage of $100 \mathrm{kV}$.

In situ reactor. The reactor and measurement cell (Supplementary Fig. 1) is composed of a PEEK reaction container inserted into a heated brass housing and surrounded by thermally insulating alumina bricks. The container is sealed by a PEEK cap pressed against the top side of the container. A 0.2 -mm-thin PEEK wall on one side of the container serves as the entrance and exit window for fluorescence-detected X-ray absorption spectroscopy. For total scattering measurements, a cylindrical quartz capillary with a diameter of $6.5 \mathrm{~mm}$ and a wall thickness of $0.5 \mathrm{~mm}$ is held in place by a PEEK support not in contact with the primary X-ray beam in order to avoid background signal from the semicrystalline PEEK. No stirring is applied to the reaction solution.

X-ray techniques. HERFD-XANES: Spectra were recorded at beamline ID26 at the European Synchrotron Radiation Facility (ESRF), Grenoble, France. Using a Si (111) double-crystal monochromator (DCM), the incident energy was varied from 7.70 to $7.78 \mathrm{keV}$ over the Co K edge. The maximum of the Co $\mathrm{Ka}_{1}$ (1s2p) fluorescence line was selected with an emission spectrometer in Rowland geometry with five $\mathrm{Si}$ (531) analyzer crystals aligned at the Bragg angle of $77^{\circ}$. HERFD-XANES spectra were recorded in continuous scan mode every $80 \mathrm{~s}$ with an overall energy resolution of $1.22 \mathrm{eV}$ and average energy steps of $0.05 \mathrm{eV}$. The position of the beam 
on the in situ cell was changed after each scan to avoid beam damage. The $\mathrm{CoO}$ reference was measured as a dry powder using a $\mathrm{Si}(311) \mathrm{DCM}$ and the $\mathrm{Co}(\mathrm{acac})_{2}$ reference was measured at beamline BM14 at ESRF selecting the $\mathrm{K} \beta_{1,3}$ (1s3p) emission line with one $\mathrm{Ge}(444)$ analyzer crystal aligned at the Bragg angle of $83^{\circ 83}$. Spectra were later aligned to the absorption edge to account for possibly different energy calibrations.

Total X-ray scattering: Data were taken at beamline P21.1 of PETRA III at Deutsches Elektronen-Synchrotron (DESY), Hamburg, Germany. Diffraction patterns were recorded every $10 \mathrm{~s}$ at an X-ray energy of $102.92 \mathrm{keV}(\lambda=0.121 \AA)$ using a digital X-ray flat-panel detector XRD1621 (Perkin Elmer Inc., USA) with a pixel size of $200 \times 200 \mu \mathrm{m}^{2}$ and a sample-to-detector distance of $0.411 \mathrm{~m}$, obtained from a calibration with a $\mathrm{CeO}_{2}$ powder standard packed into the quartz capillary of the in situ cell.

SAXS: Data were obtained at beamline P03 at PETRA III ${ }^{84}$. The X-ray energy was $12.9 \mathrm{keV}(\lambda=0.961 \AA)$. Samples were loaded in $2 \mathrm{~mm}$ quartz glass capillaries (Hilgenberg, Malsfeld, Germany). Data were recorded with a Pilatus $1 \mathrm{M}$ detector (Dectris, Baden-Dättwil, Switzerland) for $5 \times 0.1 \mathrm{~s}$ at $5.2 \mathrm{~m}$ and for $0.5 \mathrm{~s}$ at $2.0 \mathrm{~m}$ sample-to-detector distance, and combined. Additional SAXS data were recorded at a sealed tube Mo anode microfocus X-ray setup at LMU, Munich ${ }^{85}$. A Pilatus $300 \mathrm{~K}$ detector (Dectris, Baden-Dättwil, Switzerland) was used at $1.0 \mathrm{~m}$ sample-todetector distance. Samples were loaded in custom-made chambers with Kapton foils (DuPont, USA) as windows and measured for $5 \times 20 \mathrm{~min}$ and $7 \times 20 \mathrm{~min}$ before taking the median, for the samples after 20 and 90 min of reaction time, respectively.

PXRD: Data were recorded using the laboratory molybdenum anode microfocus X-ray setup at LMU, Munich ${ }^{85}$. The X-ray energy was $17.4 \mathrm{keV}(\lambda=$ $0.71 \AA$ A). Samples were dried for three days on Kapton foils (DuPont, USA) for measurements. A Pilatus $100 \mathrm{~K}$ detector (Dectris, Baden-Dättwil, Switzerland) was raster-scanned perpendicular to the beam to enlarge the $q$ range. The total exposure time per sample was $18 \mathrm{~h}$. $\mathrm{A} \mathrm{LaB}_{6}$ powder standard was used for calibration of the sample-to-detector distance and the instrumental resolution

Data analysis. HERFD-XANES: Data reduction of HERFD-XANES spectra in the energy range from 7.705 to $7.780 \mathrm{keV}$ was performed with the software package PyMCA ${ }^{86}$. Binning was applied to reduce the number of data points by a factor of five and the edge step was normalized by fitting a constant to the pre- and postedge regions. Edge positions were calculated and spectra were aligned with Athena, part of the Demeter software package ${ }^{87}$. The in situ data set was analyzed with the MCR-ALS method implemented in MATLAB (The MathWorks Inc., USA) ${ }^{59,60,88}$. An uncertainty estimation with a noise level of $3 \%$ was applied. The number of components was determined by means of singular value decomposition and the initial spectra and concentration profiles were estimated by the purest variables detection method. The ALS algorithm was applied with the following constraints: (1) non-negativity of spectra and concentrations, (2) unimodality of concentrations with $20 \%$ tolerance, and (3) convergence criterion of 0.1 .

FEFF: Theoretical XANES spectra were calculated using the FEFF code ${ }^{61}$ in version 9.6.4 with the Hedin-Lundquist energy-dependent exchange correlation potential. The settings are listed in Supplementary Table 3. For the $\mathrm{Co}_{4}(\mathrm{acac})_{8}$ tetramer, calculations with each of the Co ions set as the absorber were averaged, since their environments are not equivalent. For additional details, refer to Supplementary Notes 4 . Experimental and theoretical spectra were aligned with Athena ${ }^{87}$.

Total scattering: The azimuthal integration of the $2 \mathrm{D}$ diffraction patterns was performed with pyFAI ${ }^{89} . \mathrm{CeO}_{2}$ powder packed into the quartz capillary was used as a calibrant for the integration and the instrumental parameters in real space. The integrated data were normalized and further processed using the program xPDFsuite ${ }^{90}$ with PDFgetX $3^{91}$ to perform the background subtraction, normalization to the atomic form factors, and Fourier transformation to obtain the PDF $(\mathrm{G}(r))$. For the background subtraction, the glass capillary filled with $\mathrm{BnOH}$ was measured under the same conditions as for the synthesis. $Q_{\min }$ and $Q_{\max }$ parameters were set to $0.59 \AA^{-1}$ and $16.5 \AA^{-1}$, respectively. The resulting PDFs were sequentially refined applying PDFgui ${ }^{22}$. $Q_{\text {damp }}$ and $Q_{\text {broad }}$ were determined as 0.0463 and $0.0574 \AA^{-1}$, respectively, from the $\mathrm{CeO}_{2}$ calibrant. Structural refinements were performed using crystallographic data of cubic $\mathrm{CoO}$ ( $F m-3 m$ (225) space group) from the Inorganic Crystal Structure Database (ICSD)-9865 75

SAXS and PXRD: Intensities were azimuthally averaged and binned using the Nika ${ }^{93}$ package for Igor Pro (Wavemetrics, Portland OR, USA). A solution of $\mathrm{Co}(\mathrm{acac})_{3}$ in $\mathrm{BnOH}$ was recorded as background and subtracted from all SAXS data of ex situ samples of the synthesis. A Kapton foil background was recorded and subtracted from the PXRD data. For additional details, refer to Supplementary Notes 6 and 7.

\section{Data availability}

The SEM, TEM, HERFD-XANES, total X-ray scattering, PXRD and SAXS datasets generated and analyzed during the current study are available in the zenodo repository, https://doi.org/10.5281/zenodo.474634994. Supplementary Figures 1-21, Supplementary Tables 1-5 provide TEM and HR-TEM images, details on MCR-ALS method, HERFDXANES self-absorption correction, reaction kinetics, FEFF simulations, SAXS modeling and PXRD analysis, additional total scattering and PDF data.
Received: 27 January 2021; Accepted: 24 June 2021;

Published online: 20 July 2021

\section{References}

1. LaMer, V. K. \& Dinegar, R. H. Theory, production and mechanism of formation of monodisperse hydrosols. J. Am. Chem. Soc. 72, 4847-4854 (1950).

2. Hessel, C. M. et al. Copper selenide nanocrystals for photothermal therapy. Nano Lett. 11, 2560-2566 (2011).

3. Talapin, D. V., Lee, J.-S., Kovalenko, M. V. \& Shevchenko, E. V. Prospects of colloidal nanocrystals for electronic and optoelectronic applications. Chem. Rev. 110, 389-458 (2010).

4. Barbosa, S. et al. Tuning size and sensing properties in colloidal gold nanostars. Langmuir 26, 14943-14950 (2010).

5. Kovalenko, M. V., Scheele, M. \& Talapin, D. V. Colloidal nanocrystals with molecular metal chalcogenide surface ligands. Science 324, 1417-1420 (2009).

6. Peng, X. et al. Shape control of CdSe nanocrystals. Nature 404, 59-61 (2000)

7. Holmes, J. D., Johnston, K. P., Doty, R. C. \& Korgel, B. A. Control of thickness and orientation of solution-grown silicon nanowires. Science 287, 1471-1473 (2000).

8. Lee, J., Yang, J., Kwon, S. G. \& Hyeon, T. Nonclassical nucleation and growth of inorganic nanoparticles. Nat. Rev. Mater. 1, 16034 (2016).

9. Niederberger, M., Garnweitner, G., Ba, J., Polleux, J. \& Pinna, N. Nonaqueous synthesis, assembly and formation of metal nanocrystals. Int. J. Nanotechnol. 4, 263-272 (2007)

10. Jehannin, M., Rao, A. \& Colfen, H. New horizons of nonclassical crystallization. J. Am. Chem. Soc. 141, 10120-10136 (2019).

11. Shevchenko, E. V. et al. Colloidal synthesis and self-assembly of CoPt3 nanocrystals. J. Am. Chem. Soc. 124, 11480-11485 (2002).

12. Gebauer, D. \& Wolf, S. E. Designing solid materials from their solute state: a shift in paradigms toward a holistic approach in functional materials chemistry. J. Am. Chem. Soc. 141, 4490-4504 (2019).

13. De Yoreo, J. J. et al. Crystallization by particle attachment in synthetic, biogenic, and geologic environments. Science 349, aaa6760 (2015).

14. Schliehe, C. et al. Ultrathin PbS sheets by two-dimensional oriented attachment. Science 329, 550-553 (2010).

15. Yang, J., Son, J. S., Yu, J. H., Joo, J. \& Hyeon, T. Advances in the colloidal synthesis of two-dimensional semiconductor nanoribbons. Chem. Mater. 25, 1190-1198 (2013).

16. Ruiz-Agudo, E. et al. A non-classical view on calcium oxalate precipitation and the role of citrate. Nat. Commun. 8, 768 (2017).

17. Bai, J. et al. Ultrathin rhodium oxide nanosheet nanoassemblies: synthesis, morphological stability, and electrocatalytic application. ACS Appl. Mater. Interfaces 9, 17195-17200 (2017).

18. Litwinowicz, A.-A., Takami, S., Hojo, D., Aoki, N. \& Adschiri, T. Hydrothermal synthesis of cerium oxide nanoassemblies through coordination programming with amino acids. Chem. Lett. 43, 1343-1345 (2014).

19. Carcouet, C. C. et al. Nucleation and growth of monodisperse silica nanoparticles. Nano Lett. 14, 1433-1438 (2014).

20. Barick, K. C., Aslam, M., Prasad, P. V., Dravid, V. P. \& Bahadur, D. Nanoscale assembly of amine functionalized colloidal iron oxide. J. Magn. Magn. Mater. 321, 1529-1532 (2009).

21. He, X. et al. Phase- and size-dependent optical and magnetic properties of CoO nanoparticles. J. Phys. Chem. C 119, 9550-9559 (2015).

22. Lagunas, A., Mairata i Payeras, A., Jimeno, C., Puntes, V. F. \& Pericàs, M. A. Low-temperature synthesis of $\mathrm{CoO}$ nanoparticles via chemically assisted oxidative decarbonylation. Chem. Mater. 20, 92-100 (2008).

23. Staniuk, M. et al. Matching the organic and inorganic counterparts during nucleation and growth of copper-based nanoparticles-in situ spectroscopic studies. CrystEngComm 17, 6962-6971 (2015).

24. Kränzlin, N., van Beek, W., Niederberger, M. \& Koziej, D. Mechanistic studies as a tool for the design of copper-based heterostructures. Adv. Mater. Interfaces 2, 1500094 (2015).

25. Staniuk, M. et al. Puzzling mechanism behind a simple synthesis of cobalt and cobalt oxide nanoparticles: In situ synchrotron X-ray absorption and diffraction studies. Chem. Mater. 26, 2086-2094 (2014).

26. Kränzlin, N. et al. Rationale for the crystallization of titania polymorphs in solution. Nanoscale 6, 14716-14723 (2014).

27. Hirsch, $\mathrm{O}$. et al. Aliovalent $\mathrm{Ni}$ in $\mathrm{MoO} 2$ lattice-probing the structure and valence of $\mathrm{Ni}$ and its implication on the electrochemical performance. Chem. Mater. 26, 4505-4513 (2014).

28. Koziej, D. et al. Interplay between size and crystal structure of molybdenum dioxide nanoparticles-synthesis, growth mechanism, and electrochemical performance. Small 7, 377-387 (2011). 
29. Hubner, M. et al. The structure and behavior of platinum in $\mathrm{SnO} 2$-based sensors under working conditions. Angew. Chem. Int. Ed. Engl. 50, 2841-2844 (2011).

30. Strach, M. et al. Insights into reaction intermediates to predict synthetic pathways for shape-controlled metal nanocrystals. J. Am. Chem. Soc. 141, 16312-16322 (2019).

31. Hutchings, G. S. et al. In situ formation of cobalt oxide nanocubanes as efficient oxygen evolution catalysts. J. Am. Chem. Soc. 137, 4223-4229 (2015).

32. Kvashnina, K. O. et al. A novel metastable pentavalent plutonium solid phase on the pathway from aqueous plutonium(VI) to $\mathrm{PuO} 2$ nanoparticles. Angew. Chem. Int. Ed. Engl. 58, 17558-17562 (2019).

33. Hirsch, O. et al. High-energy resolution X-ray absorption and emission spectroscopy reveals insight into unique selectivity of La-based nanoparticles for $\mathrm{CO}(2)$. PNAS 112, 15803-15808 (2015).

34. Glatze, P., Singh, J., Kvashnina, K. O. \& van Bokhoven, J. A. In situ characterization of the $5 \mathrm{~d}$ density of states of Pt nanoparticles upon adsorption of CO. J. Am. Chem. Soc. 132, 2555-2557 (2010).

35. Kuciakowski, J. et al. Selective magnetometry of superparamagnetic iron oxide nanoparticles in liquids. Nanoscale 12, 16420-16426 (2020).

36. Saha, D., Bøjesen, E. D., Mamakhel, A. H., Bremholm, M. \& Iversen, B. B. In situ PDF study of the nucleation and growth of intermetallic PtPb nanocrystals. ChemNanoMat 3, 472-478 (2017).

37. Tyrsted, C. et al. Evolution of atomic structure during nanoparticle formation. IUCrJ 1, 165-171 (2014)

38. Jensen, K. M. et al. Revealing the mechanisms behind $\mathrm{SnO} 2$ nanoparticle formation and growth during hydrothermal synthesis: an in situ total scattering study. J. Am. Chem. Soc. 134, 6785-6792 (2012).

39. Zobel, M., Neder, R. B. \& Kimber, S. A. J. Universal solvent restructuring induced by colloidal nanoparticles. Science 347, 292-294 (2015).

40. Narayanan, T., Wacklin, H., Konovalov, O. \& Lund, R. Recent applications of synchrotron radiation and neutrons in the study of soft matter. Crystallogr. Rev. 23, 160-226 (2017).

41. Ingham, B. X-ray scattering characterisation of nanoparticles. Crystallogr. Rev. 21, 229-303 (2015).

42. Montanarella, F. et al. Crystallization of nanocrystals in spherical confinement probed by in situ X-ray scattering. Nano Lett. 18, 3675-3681 (2018).

43. Zheng, X. et al. Self-templated synthesis of microporous $\mathrm{CoO}$ nanoparticles with highly enhanced performance for both photocatalysis and lithium-ion batteries. J. Mater. Chem. A 1, 1394-1400 (2013).

44. Qi, Y., Zhang, H., Du, N. \& Yang, D. Highly loaded CoO/graphene nanocomposites as lithium-ion anodes with superior reversible capacity. J. Mater. Chem. A 1, 2337 (2013).

45. Sun, B., Liu, H., Munroe, P., Ahn, H. \& Wang, G. Nanocomposites of CoO and a mesoporous carbon (CMK-3) as a high performance cathode catalyst for lithium-oxygen batteries. Nano Res. 5, 460-469 (2012).

46. Jiang, Z. J. \& Jiang, Z. Interaction induced high catalytic activities of $\mathrm{CoO}$ nanoparticles grown on nitrogen-doped hollow graphene microspheres for oxygen reduction and evolution reactions. Sci. Rep. 6, 27081 (2016).

47. Guo, S., Zhang, S., Wu, L. \& Sun, S. Co/CoO nanoparticles assembled on graphene for electrochemical reduction of oxygen. Angew. Chem. Int. Ed. Engl. 51, 11770-11773 (2012).

48. Liao, L. et al. Efficient solar water-splitting using a nanocrystalline $\mathrm{CoO}$ photocatalyst. Nat. Nanotechnol. 9, 69-73 (2014).

49. Park, K.-W. \& Kolpak, A. M. Understanding photocatalytic overall water splitting on $\mathrm{CoO}$ nanoparticles: effects of facets, surface stoichiometry, and the $\mathrm{CoO} /$ water interface. J. Catal. 365, 115-124 (2018)

50. Li, Y. et al. Morphology-dominant microwave absorption enhancement and electron tomography characterization of $\mathrm{CoO}$ self-assembly $3 \mathrm{D}$ nano-flowers. J. Mater. Chem. C 2, 5216 (2014).

51. Rai, A. K., Anh, L. T., Gim, J. \& Kim, J. One-step synthesis of CoO anode material for rechargeable lithium-ion batteries. Ceram. Int. 39, 9325-9330 (2013).

52. Chen, Z., Xu, A., Zhang, Y. \& Gu, N. Preparation of $\mathrm{NiO}$ and $\mathrm{CoO}$ nanoparticles using $\mathrm{M} 2+$-oleate $(\mathrm{M}=\mathrm{Ni}, \mathrm{Co})$ as precursor. Curr. Appl. Phys. 10, 967-970 (2010).

53. Niederberger, M. Nonaqueous sol-gel routes to metal oxide nanoparticles. Acc. Chem. Res. 40, 793-800 (2007).

54. Papaefthimiou, V. et al. Nontrivial redox behavior of nanosized cobalt: new insights from ambient pressure X-ray photoelectron and absorption spectroscopies. ACS Nano 5, 2182-2190 (2011).

55. de Groot, F., Vanko, G. \& Glatzel, P. The 1s X-ray absorption pre-edge structures in transition metal oxides. J. Phys. Condens. Matter 21, 104207 (2009).

56. Choi, H. C. et al. Local structural characterization for electrochemical insertion-extraction of lithium into $\mathrm{CoO}$ with $\mathrm{X}$-ray absorption spectroscopy. J. Phys. Chem. B 106, 9252-9260 (2002).

57. Sankar, G., Sarode, P. R. \& Rao, C. N. R. A XANES study of mixed-valence transition-metal oxides and rare-earth alloys. Chem. Phys. 76, 435-442 (1983).
58. Andonova, S., de Ávila, C. N., Arishtirova, K., Bueno, J. M. C. \& Damyanova, $\mathrm{S}$. Structure and redox properties of Co promoted $\mathrm{Ni} / \mathrm{Al} 2 \mathrm{O} 3$ catalysts for oxidative steam reforming of ethanol. Appl. Catal. B 105, 346-360 (2011).

59. de Juan, A., Jaumot, J. \& Tauler, R. Multivariate curve resolution (MCR). Solving the mixture analysis problem. Anal. Methods 6, 4964-4976 (2014)

60. Jaumot, J., Gargallo, R., de Juan, A. \& Tauler, R. A graphical user-friendly interface for MCR-ALS: a new tool for multivariate curve resolution in MATLAB. Chemometr. Intell. Lab. Syst. 76, 101-110 (2005).

61. Rehr, J. J., Kas, J. J., Vila, F. D., Prangebc, M. P. \& Jorissena, K. Parameter-free calculations of X-ray spectra with FEFF9. Phys. Chem. Chem. Phys. 12, 5503-5513 (2010).

62. Radón, M., Srebro, M. \& Broclawik, E. Conformational stability and spin states of cobalt(II) acetylacetonate: CASPT2 and DFT study. J. Chem. Theory Comput. 5, 1237-1244 (2009).

63. von Chrzanowski, L. S., Lutz, M. \& Spek, A. L. alpha-Tris(2,4-pentanedionatokappa(2)O,O')cobalt(III) at 240, 210, 180, 150 and 110 K. Acta Crystallogr. C 63, m283-m228 (2007).

64. Niwa, H. et al. High-energy-resolution XANES of layered oxides for sodiumion battery. Appl. Phys. Express 12, 052005 (2019).

65. Sano, M. XANES study at the Co K absorption edge in a series of cobalt(III) complexes. Inorg. Chem. 27, 4249-4253 (1988).

66. Saib, A. M., Borgna, A., van de Lossdrecht, J., van Berge, P. J. \& Niemantsverdriet, J. W. XANES study of the susceptibility of nano-sized cobalt crystallites to oxidation during realistic Fischer-Tropsch synthesis. Appl. Catal. A 312, 12-19 (2006).

67. Vråstad, T. et al. Interfacial chemistry of cobalt(II) during sol-gel synthesis of cobalt-containing mesoporous materials. Microporous Mesoporous Mater. 80, 291-300 (2005).

68. Choy, J. H., Jung, H. \& Yoon, J. B. Co K-edge XAS study on a new cobaltdoped-SiO2 pillared clay. J. Synchrotron Radiat. 8, 599-601 (2001).

69. Booth, C. H. \& Bridges, F. Improved self-absorption correction for fluorescence measurements of extended X-ray absorption fine-structure. Phys. Scr. T115, 202-204 (2005).

70. Vreshch, V. D., Yang, J. H., Zhang, H., Filatov, A. S. \& Dikarev, E. V. Monomeric square-planar cobalt(II) acetylacetonate: mystery or mistake? Inorg. Chem. 49, 8430-8434 (2010).

71. Cotton, F. A. \& Elder, R. C. Crystal structure of tetrameric cobalt(II) acetylacetonate. Inorg. Chem. 4, 1145-1151 (1965).

72. Ludi, B., Suess, M. J., Werner, I. A. \& Niederberger, M. Mechanistic aspects of molecular formation and crystallization of zinc oxide nanoparticles in benzyl alcohol. Nanoscale 4, 1982-1995 (2012).

73. Bilecka, I., Elser, P. \& Niederberger, M. Kinetic and thermodynamic aspects in the microwave-assisted synthesis of $\mathrm{ZnO}$ nanoparticles in benzyl alcohol. ACS Nano 3, 467-477 (2009).

74. Burgess, J., Fawcett, J. \& Russell, D. R. Bis(2,4-pentanedionato)cobalt(II). Acta Crystallogr. C 67, e13-e13 (2000)

75. Sasaki, S., Fujino, K. \& Tacéuchi, Y. X-ray determination of electron density distributions in oxides, $\mathrm{MgO}, \mathrm{MnO}, \mathrm{CoO}$, and $\mathrm{NiO}$, and atomic scattering factors of their constituent atoms. Proc. Jpn. Acad. B 55, 43-48 (1979).

76. Momma, K. \& Izumi, F. VESTA 3 for three-dimensional visualization of crystal, volumetric and morphology data. J. Appl. Crystallogr. 44, 1272-1276 (2011).

77. Mildner, D. F. R. \& Hall, P. L. Small-angle scattering from porous solids with fractal geometry. J. Phys. D Appl. Phys. 19, 1535-1545 (1986).

78. Glatter, O. \& Kratky, O. Small Angle X-Ray Scattering (Academic Press, 1982).

79. Kapuscinski, M. et al. Temporal evolution of superlattice contraction and defect-induced strain anisotropy in mesocrystals during nanocube selfassembly. ACS Nano 14, 5337-5347 (2020).

80. Lv, Z. P., Kapuscinski, M. \& Bergstrom, L. Tunable assembly of truncated nanocubes by evaporation-driven poor-solvent enrichment. Nat. Commun. 10, 4228 (2019).

81. Josten, E. et al. Superlattice growth and rearrangement during evaporationinduced nanoparticle self-assembly. Sci. Rep. 7, 2802 (2017).

82. Tiede, D. M., Kwon, G., He, X., Mulfort, K. L. \& Martinson, A. B. F. Characterizing electronic and atomic structures for amorphous and molecular metal oxide catalysts at functional interfaces by combining soft X-ray spectroscopy and high-energy X-ray scattering. Nanoscale 12, 13276-13296 (2020).

83. Kvashnina, K. O. \& Scheinost, A. C. A Johann-type X-ray emission spectrometer at the Rossendorf beamline. J. Synchrotron Radiat. 23, 836-841 (2016).

84. Buffet, A. et al. P03, the microfocus and nanofocus X-ray scattering (MiNaXS) beamline of the PETRA III storage ring: the microfocus endstation. J. Synchrotron Radiat. 19, 647-653 (2012).

85. Bruetzel, L. K. et al. A Mo-anode-based in-house source for small-angle X-ray scattering measurements of biological macromolecules. Rev. Sci. Instrum. 87, 025103 (2016). 
86. Solé, V. A., Papillon, E., Cotte, M., Walter, P. \& Susini, J. A multiplatform code for the analysis of energy-dispersive X-ray fluorescence spectra. Spectrochimica Acta Part B: At. Spectrosc. 62, 63-68 (2007).

87. Ravel, B. \& Newville, M. ATHENA, ARTEMIS, HEPHAESTUS: data analysis for X-ray absorption spectroscopy using IFEFFIT. J. Synchrotron Radiat. 12, 537-541 (2005).

88. Jaumot, J., de Juan, A. \& Tauler, R. MCR-ALS GUI 2.0: new features and applications. Chemometr. Intell. Lab. Syst. 140, 1-12 (2015).

89. Ashiotis, G. et al. The fast azimuthal integration Python library: pyFAI. $J$. Appl. Crystallogr. 48, 510-519 (2015).

90. Yang, X., Juhás, P., Farrowa, C. L. \& Billinge, S. J. L. xPDFsuite: an end-to-end software solution for high throughput pair distribution function transformation, visualization and analysis. ArXiv 1402, 3163 (2014).

91. Juhás, P., Davis, T., Farrow, C. L. \& Billinge, S. J. L. PDFgetX3: a rapid and highly automatable program for processing powder diffraction data into total scattering pair distribution functions. J. Appl. Crystallogr. 46, 560-566 (2013).

92. Farrow, C. L. et al. PDFfit2 and PDFgui: computer programs for studying nanostructure in crystals. J. Phys. Condens. Matter 19, 335219 (2007).

93. Ilavsky, J. Nika: software for two-dimensional data reduction. J. Appl. Crystallogr. 45, 324-328 (2012).

94. Grote, L. et al. Raw data for "X-ray studies bridge the molecular and macro length scales during the emergence of $\mathrm{CoO}$ assemblies". zenodo https://doi. org/10.5281/zenodo.4746349 (2021).

\section{Acknowledgements}

This work was supported by the European Research Council within the project LINCHPIN (grant no. 818941), by the Bundesministerium für Bildung und Forschung (BMBF) via project LUCENT (grant no. 05K19WMA), by the Bavarian State Ministry of Science, Research, and Arts through the grant "SolarTechnologies go Hybrid (SolTech)", by the Cluster of Excellence "The Hamburg Centre for Ultrafast Imaging" of the Deutsche Forschungsgemeinschaft (DFG) - EXC 1074_-project ID 194651731, by the Cluster of Excellence "CUI: Advanced Imaging of Matter" of the DFG-EXC 2056project ID 390715994, by the DFG via project ID 201269156-SFB1032, and by the São Paulo Research Foundation (FAPESP, grant no. 2018/08271-7 and 2016/25267-8). This work benefited from the use of the SasView application (version 4.2.2), originally developed under NSF award DMR-0520547, further developed within the European Union's Horizon 2020 research and innovation programme under the SINE2020 project (grant no. 654000). We thank Dr. Lucia Amidani, Dr. Rafal Baran, Dr. Roelof van Silfhout, Dirk Detollenaere, Florian Ledrappier, Martina Ober, and Ludwig Hendl for supporting the X-ray measurements and laboratory experiments, Andreas Kornowski, Andrea Koeppen, Stefan Werner and Susanne Kempter for TEM imaging, Tarcísio Perfecto for designing Fig. 7 and ESRF, ID26 for beamtime allocation. We acknowledge DESY (Hamburg, Germany), a member of the Helmholtz Association HGF, for the provision of experimental facilities. Parts of this research were carried out at PETRA III and we would like to thank Prof. Stephan Roth, Dr. Matthias Schwarzkopf, and Dr. Martin Roelsgaard for assistance in using beamlines P03 and P21.1.

\section{Author contributions}

D.K., L.G. and C.A.Z. conceived the project. L.G., C.A.Z., D.K., K.P., K.O.K. and S.B. conducted the X-ray absorption experiments. L.G., C.A.Z., M.R. and S.Y.H.-M. designed and conducted the X-ray total scattering experiments. K.F., P.R., B.N., and P.P. designed and conducted the SAXS experiments. A.-C.D., B.D., and O.I. supported the synchrotron experiments. L.G. and K.P. analyzed the HERFD-XANES data. C.A.Z., and A.-C.D. analyzed the total scattering data. K.F. and P.R. analyzed the SAXS data. All authors contributed to data interpretation and the preparation of the paper.

\section{Funding}

Open Access funding enabled and organized by Projekt DEAL.

\section{Competing interests}

The authors declare no competing interest.

\section{Additional information}

Supplementary information The online version contains supplementary material available at https://doi.org/10.1038/s41467-021-24557-z.

Correspondence and requests for materials should be addressed to D.K.

Peer review information Nature Communications thanks Lennart Bergström, Karl Mandel, and other, anonymous, reviewers for their contributions to the peer review of this work.

Reprints and permission information is available at http://www.nature.com/reprints Publisher's note Springer Nature remains neutral with regard to jurisdictional claims in published maps and institutional affiliations.

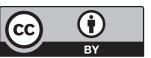

Open Access This article is licensed under a Creative Commons Attribution 4.0 International License, which permits use, sharing, adaptation, distribution and reproduction in any medium or format, as long as you give appropriate credit to the original author(s) and the source, provide a link to the Creative Commons license, and indicate if changes were made. The images or other third party material in this article are included in the article's Creative Commons license, unless indicated otherwise in a credit line to the material. If material is not included in the article's Creative Commons license and your intended use is not permitted by statutory regulation or exceeds the permitted use, you will need to obtain permission directly from the copyright holder. To view a copy of this license, visit http://creativecommons.org/ licenses/by/4.0/.

(C) The Author(s) 2021 